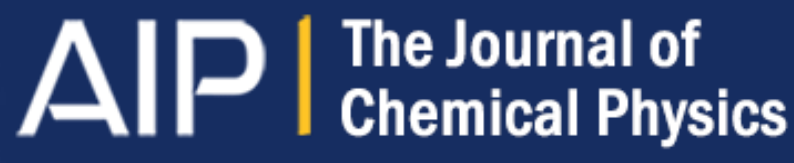

\section{High order finite difference algorithms for solving the Schrödinger equation in molecular dynamics. II. Periodic variables}

Raul Guantes and Stavros C. Farantos

Citation: The Journal of Chemical Physics 113, 10429 (2000); doi: 10.1063/1.1324004

View online: http://dx.doi.org/10.1063/1.1324004

View Table of Contents: http://scitation.aip.org/content/aip/journal/jcp/113/23?ver=pdfcov

Published by the AIP Publishing

\section{Articles you may be interested in}

Finite difference method for solving the Schrödinger equation with band nonparabolicity in mid-infrared quantum cascade lasers

J. Appl. Phys. 108, 113109 (2010); 10.1063/1.3512981

A Parallel Code for Solving the Molecular Time Dependent Schrödinger Equation in Cartesian Coordinates AIP Conf. Proc. 1148, 241 (2009); 10.1063/1.3225285

Response to "Comment on "High order finite difference algorithms for solving the Schrödinger equation in molecular dynamics'” [J. Chem. Phys. 115, 6794 (2001)]

J. Chem. Phys. 115, 6796 (2001); 10.1063/1.1400784

Comment on "High order finite difference algorithms for solving the Schrödinger equation in molecular dynamics"

[J. Chem. Phys. 111, 10827 (1999)]

J. Chem. Phys. 115, 6794 (2001); 10.1063/1.1400783

High order finite difference algorithms for solving the Schrödinger equation in molecular dynamics

J. Chem. Phys. 111, 10827 (1999); 10.1063/1.480446

\section{AlP Re-register for Table of Content Alerts}




\title{
High order finite difference algorithms for solving the Schrödinger equation in molecular dynamics. II. Periodic variables
}

\author{
Raul Guantes ${ }^{\text {a) }}$ and Stavros C. Farantos ${ }^{\text {b) }}$ \\ Institute of Electronic Structure and Laser, Foundation for Research and Technology-Hellas, \\ Post Office Box 1527, Iraklion 71110, Crete, Greece
}

(Received 10 May 2000; accepted 20 September 2000)

\begin{abstract}
Variable high order finite difference methods are applied to calculate the action of molecular Hamiltonians on the wave function using centered equi-spaced stencils, mixed centered and one-sided stencils, and periodic Chebyshev and Legendre grids for the angular variables. Results from one-dimensional model Hamiltonians and the three-dimensional spectroscopic potential of $\mathrm{SO}_{2}$ demonstrate that as the order of finite difference approximations of the derivatives increases the accuracy of pseudospectral methods is approached in a regular manner. The high order limit of finite differences to Fourier and general orthogonal polynomial discrete variable representation methods is analytically and numerically investigated. (C) 2000 American Institute of Physics.
\end{abstract}

[S0021-9606(00)00247-6]

\section{INTRODUCTION}

In a previous article, ${ }^{1}$ herein referred as Paper I, we reported results from the application of a variable order finite difference (FD) method to approximate the action of a Hamiltonian operator on the wave function in the timedependent Schrödinger equation or the Hamiltonian matrix elements in the time-independent picture. One-, two-, and three-dimensional model potentials in Cartesian and radial coordinates were used to investigate the accuracy and the stability of these methods, whereas in a companion paper, ${ }^{2}$ the time-dependent Schrödinger equation was solved for the van der Waals system $\mathrm{Ar}_{3}$. The impetus for this project was given by recent advances in high order finite difference approximations. We mainly refer to the limit of infinite order finite difference formulas with respect to global pseudospectral methods (PS) investigated by Fornberg, ${ }^{3}$ and Boyd's work which views finite difference methods as a certain sum acceleration of pseudospectral techniques. ${ }^{4}$

Finite difference approximations of the derivatives of a function $F(x)$ can be extracted by interpolating $F(x)$ with Lagrange polynomials, $P(x)$. This allows one to calculate the derivatives analytically at arbitrarily chosen grid points and with a variable order of approximation. The Lagrange fundamental polynomials of order $N-1$ are defined by

$$
L_{k}(x)=\prod_{j=1}^{N}{ }^{\prime}\left(x-x_{j}\right) / \prod_{j=1}^{N}{ }^{\prime}\left(x_{k}-x_{j}\right), \quad k=1,2, \ldots, N,
$$

where the prime means that the term $j=k$ is not included in

\footnotetext{
${ }^{a)}$ Current address: Instituto de Matematicas y Fisica Fundamental, Consejo Superior de Investigaciones Cientificas, Serrano 123, 28006 Madrid, Spain.

${ }^{b)}$ Also at Department of Chemistry, University of Crete, Iraklion 711 10, Crete, Greece.
}

the products. The values of $L_{k}\left(x_{j}\right)$ are zero for $j \neq k$ and one for $j=k$ by construction. The function can then be approximated as

$$
F(x) \approx P_{N}(x)=\sum_{k=1}^{N} F\left(x_{k}\right) L_{k}(x) .
$$

$P_{N}$ is a polynomial of order $N-1$. In Paper I we discussed how FD is related to the sinc-DVR method by taking the limit in the two above-mentioned senses.

(1) An infinite order limit of centered FD formulas on an equispaced grid yields the discrete variable representation (DVR) result when we use as a basis set the sinc functions $[\operatorname{Sinc}(x) \equiv \sin (\pi x) / \pi x] ., 5$ Although, this limit is defined formally as $N$, the number of grid points used in the approximation, tends to infinity, some theoretical considerations ${ }^{3}$ as well as numerical results ${ }^{1}$ lead us to expect that the accuracy of the FD approximation is the same to that of the DVR method as we approach the full grid to calculate the FD coefficients.

(2) FD can also be viewed as a sum acceleration method which improves the convergence of the pseudospectral approximation. ${ }^{4}$ The rate of convergence is, however, nonuniform in the wave number, giving very high accuracy for low wave numbers and poor accuracy for wave numbers near the aliasing limit. ${ }^{6}$ However, this does not cause a severe practical limitation, since, by increasing the number of grid points in the appropriate region we can have an accurate enough representation of the true spectrum in the range of interest. This is one property which makes FD useful as an alternative to the common $\mathrm{DVR}^{7}$ and other PS methods such as fast Fourier transform techniques (FFT). ${ }^{8,9}$

Thachuk and Schatz ${ }^{10}$ in their study on methods for calculating thermal rate coefficients with flux correlation functions also used high order finite difference methods. Employ- 
ing a one-dimensional symmetric Eckart potential they examined and evaluated several methods for the time propagation and the spatial derivative calculations required by the action of the Laplacian on the wave function.

Parallel to our work, Mazziotti ${ }^{11}$ has applied Boyd's sum acceleration methods on a one-dimensional Morse function. In fact, under the name spectral difference he examines four different methods; the truncated sinc, Boyd's Euler and finite difference sum acceleration methods, and the Lagrange distributed approximating functional (LDAF) approach of Kouri and co-workers. ${ }^{12}$

The current interest in finite difference methods is fully justified when solutions of the Schrödinger equation are required for multidimensional systems such as polyatomic molecules. The present most popular methods employed in quantum molecular dynamics are the fast Fourier transform and the discrete variable representation techniques. FFT generally uses hypercubic grid domains which result in wasted configuration space sampling. A large number of the selected configuration points correspond to high potential energy values, which do not contribute to the eigenstates that we are seeking. Global DVR methods allow us to choose easily the configuration points which are relevant to the states we want to calculate, but still, we must employ in each dimension all grid points. Local methods such as FD have the advantages of DVR but also produce matrices with less nonzero matrix elements provided that the PS accuracy is achieved at lower order than the high order limit.

There are some other benefits for FD with respect to global pseudospectral methods. Convergence can be examined not only by increasing the number of grid points but also by varying in a systematic way the order of approximation of the derivatives. Finite difference methods may incorporate several boundary conditions and choose the grid points without necessarily relying on specific basis functions. The topography of the multidimensional molecular potential functions is usually complex. The ability of using non equispaced grids is as important as keeping the grid points in accordance to the chosen energy interval. The computer codes for a FD representation of the Hamiltonian can be parallelized relatively easily, since the basic operation is the multiplication of a vector by a sparse matrix. Parallelization is an obligatory task when we deal with systems of more than three degrees of freedom and we look for highly excited states.

Sinc-DVR methods are appropriate for radial variables where the wave function must vanish at the edge of the grid $[\Psi(R)=0$ for $a \geqslant R \geqslant b]$. The FD weights required in approximating the derivatives of the wave function close to the borders of the grid can be calculated for this boundary condition by extending the grid intervals with fictitious points. Another type of radial coordinates frequently encountered in molecular dynamics are those which cannot be extended with fictitious points. Such a variable is the distance of an atom from the center-of-mass of a diatomic molecule in Jacobi coordinates which may start from zero for linear configuration. In this case it is necessary to employ one-sided FD formulas.

Most of vibrational Hamiltonians, however, or Hamilto- nians describing molecule-surface encounters, atomdiatom, or four-atom chemical reactions require the use of angular variables, and therefore, periodic boundary conditions. It is interesting to see if FD methods can be applied to angular variables with the same effectiveness demonstrated for radial variables and to investigate if the same limits can be approached here. In this context, it is worth studying FD approximations with different grid distributions. Doing this we can compare some local approaches to the solution of the Schrödinger equation with well established DVR methods used for angular variables, such as Legendre or Chebyshev orthogonal polynomial expansions which lead to nonuniform grids compared to the Fourier method which is based on uniform grids.

The purpose of the present article is to investigate the accuracy and stability of variable high order finite difference approximations to molecular-type Hamiltonians which usually employ curvilinear coordinates including angle variables. Uniform and nonuniform stencils are examined. The article is organized in the same fashion as Paper I. In Sec. II we extract the limit formulas for a periodic angle variable and study simple well-known model systems. We will make analytical connections between FD and the Fourier method, as well as common orthogonal polynomial DVR methods. In Sec. III we study a real triatomic molecule, the sulfur dioxide, using a spectroscopic potential in Jacobi coordinates. ${ }^{13}$ Vibrational levels of $\mathrm{SO}_{2}$ have been calculated by Guo and co-workers up to $4 \mathrm{eV}$ above the zero-point energy $(\mathrm{ZPE})^{14-16}$ with filter diagonalization techniques ${ }^{17-19}$ and DVR for the Hamiltonian. We do not compute accurate vibrational eigenenergies but we investigate the stability in time evolution of initial Gaussian wave packets. Finally, in Sec. IV we summarize the conclusions of the present and previous studies.

\section{LIMIT METHODS}

\section{A. Periodic uniform grids}

Grid representations of the Schrödinger equation can be obtained by first defining global smooth basis functions, $\phi_{j}(x)$, to expand the wave function as

$$
\Psi(x) \approx \Psi_{N}(x)=\sum_{j=1}^{N} a_{j} \phi_{j}(x) .
$$

Different global basis functions define different pseudospectral methods. From such so-called finite basis representation (FBR) we can transform to a cardinal set of basis functions, $u_{j}(x)$, or the discrete variable representation (DVR) as it is usually called, by choosing $N$ grid points, $x_{i}$, at which the wave function is calculated. The cardinal functions obey the $\delta$-Kronecker property

$$
u_{j}\left(x_{i}\right)=\delta_{i j},
$$

so that the wave function is represented in the set of grid points as

$$
\Psi_{N}(x)=\sum_{j=1}^{N} \Psi\left(x_{j}\right) u_{j}(x) .
$$


Notice, that the expansion coefficients are the exact function values at the chosen grid points.

The transformation from FBR to the cardinal basis set is unitary and the new basis is given in terms of the old one by

$$
u_{j}(x)=\sum_{i=1}^{N}\left\langle\phi_{i} \mid u_{j}\right\rangle \phi_{i}(x), \quad j=1, \ldots N .
$$

A common procedure now is to evaluate the matrix elements $\left\langle\phi_{i} \mid u_{j}\right\rangle$ by Gaussian quadrature, such that the integral becomes exact for a polynomial-type basis. A number of different approximation methods can be obtained by defining different basis functions and quadrature rules. ${ }^{20,21}$ In general, using the $\delta$-Kronecker property of $u_{j}(x)$ [Eq. (4)] we can write

$$
\left\langle\phi_{i} \mid u_{j}\right\rangle=\sum_{k=1}^{N} w_{k} \phi_{i}^{*}\left(x_{k}\right) u_{j}\left(x_{k}\right)=w_{j} \phi_{i}^{*}\left(x_{j}\right),
$$

where the grid points $x_{k}$ and the corresponding weights $w_{k}$ depend on the chosen quadrature rule. A widely used set of basis functions in the solution of the Schrödinger equation is the Fourier set

$$
\phi_{j}(x)=\frac{1}{\sqrt{2 \pi}} \exp (i 2 \pi j x / L),
$$

where $L$ is the length which defines the periodicity of the function and $j=-M, \ldots, 0, \ldots, M$. We can transform these functions to a set of cardinal functions by using a uniform grid in $x$ with an odd number of points, $N=2 M+1$, and employing Chebyshev quadrature. This results in the real trigonometric series ${ }^{3,20}$

$$
u_{j}(x)=\frac{w_{j}}{2 \pi}\left[1+2 \sum_{k=1}^{M} \cos \left(2 \pi k\left(x-x_{j}\right) / L\right)\right] .
$$

The weights are equal to $w_{j}=L / N$ for Chebyshev quadrature. Assuming for simplicity that $x$ is an angular variable with period $L=2 \pi$, and using the trigonometric identity

$$
\frac{1}{2}+\sum_{k=1}^{M} \cos (k \alpha)=\frac{\sin [(M+1 / 2) \alpha]}{2 \sin (\alpha / 2)},
$$

Eq. (9) gives the Fourier cardinal functions

$$
u_{j}(x)=\frac{\sin \left[N\left(x-x_{j}\right) / 2\right]}{N \sin \left[\left(x-x_{j}\right) / 2\right]} .
$$

The $\delta$-Kronecker property can be immediately checked, and we can derive analytically the derivatives of the wave function $\Psi(x)$ when it is expanded in the cardinal functions $u_{j}(x)$ [Eq. (5)]:

$$
\left.\frac{d^{m} \Psi(x)}{d x^{m}}\right|_{x=x_{k}}=\sum_{j=1}^{N} b_{k, j}^{(m)} \Psi\left(x_{j}\right),
$$

with $b_{k, j}^{(m)}$ the $m$ th derivative of $u_{j}(x)$ evaluated at $x=x_{k}$. In Appendix I we give the first and second derivatives of the Fourier cardinal functions, $u_{j}(x)$, for periodicities $L$ $=\pi, 2 \pi$.

We can understand the correspondence between Fourier cardinal functions and finite differences by recalling a rela- tion between the Fourier cardinal basis and the sinc basis: a Fourier cardinal basis can be seen as a sum of sinc basis functions repeated periodically with periodicity $2 \pi$, i.e.,

$$
\frac{\sin \left[N\left(x-x_{j}\right) / 2\right]}{N \sin \left[\left(x-x_{j}\right) / 2\right]}=\sum_{k=-\infty}^{k=\infty} \operatorname{sinc}\left[\left(x-x_{j}+2 \pi k\right) / \Delta x\right],
$$

$\Delta x=2 \pi / N$ in this case and we have used the fraction expansion $\pi / \sin (\pi x)=\sum_{k=-\infty}^{k=\infty}(-1)^{k} /(x+k){ }^{22}$ Since sinc functions are the infinite order limit of an equispaced FD, the correspondence now is that periodically repeated FD stencils will tend to the PS limit of Fourier functions as the number of grid points in the stencil approaches the total number of grid points in one period. Also, because equispaced FD can be considered as a robust sum acceleration scheme of a sinc function series, we expect the same convergence properties of the FD approximation to the Fourier series as the one we found for radial variables in Paper I.

To demonstrate this we used a simple model, which is however instructive since it appears in many vibrational Hamiltonians with azimuthal symmetry: a one-dimensional rigid-free rotor, represented by the Schrödinger equation $(0 \leqslant \phi<2 \pi)$,

$$
-\frac{\hbar^{2}}{2 \mu r^{2}} \frac{d^{2} \Psi}{d \phi^{2}}=E \Psi
$$

The exact eigenfunctions and eigenvalues are $\Psi_{m}(\phi)$ $=(1 / \sqrt{2 \pi}) e^{i m \phi}$ and $E=\hbar^{2} m^{2} / 2 \mu r^{2}, m=0, \pm 1, \pm 2, \ldots$, respectively. Notice that a numerical solution using Fourier functions is exact in this case, and so the pseudospectral solution with Fourier cardinal functions, since they have the form of a cosine series and the Chebyshev quadrature to evaluate the transformation matrix Eq. (7), is also exact. A FD should also converge to the exact result but faster than the cosine series.

By representing the wave function in $N_{\text {tot }}$ grid points and evaluating the second derivatives by interpolating the function with stencils of $N$-points $\left(3 \leqslant N \leqslant N_{\text {tot }}\right)$ the kinetic energy nonzero matrix elements have the form

$$
T_{k, j}=-\frac{\hbar^{2}}{2 \mu r^{2}} b_{k, j}^{(2)}, \quad 1 \leqslant k \leqslant N_{\text {tot }} \text { and }-M \leqslant j-k \leqslant M,
$$

where $N=2 M+1$.

We applied both methods, Fourier-DVR with weights $b_{k, j}^{(2)}$ given in the Appendix, and variable order FD approximations with periodic boundary conditions by choosing the grid points as

$$
\phi_{i}=\left\{\begin{array}{ll}
\phi_{N+i} & i<1, \\
\phi_{i-N} & i>N
\end{array}\right\} .
$$

The weights needed in the FD method are computed with Fornberg's algorithm. ${ }^{23}$ This is an efficient algorithm to compute any derivative, at any order and at arbitrarily spaced grid points. Details can be found in Fornberg's book. ${ }^{3}$ In Fig. 1 we show the decimal logarithm of the fractional error $\left(E_{\text {app }} / E_{\text {ex }}-1\right)$ for the eigenvalue $m=+20$ using FD approximations of increasing order (solid line). The $M$ th order approximation means that we use stencils of $N=2 M+1$ 


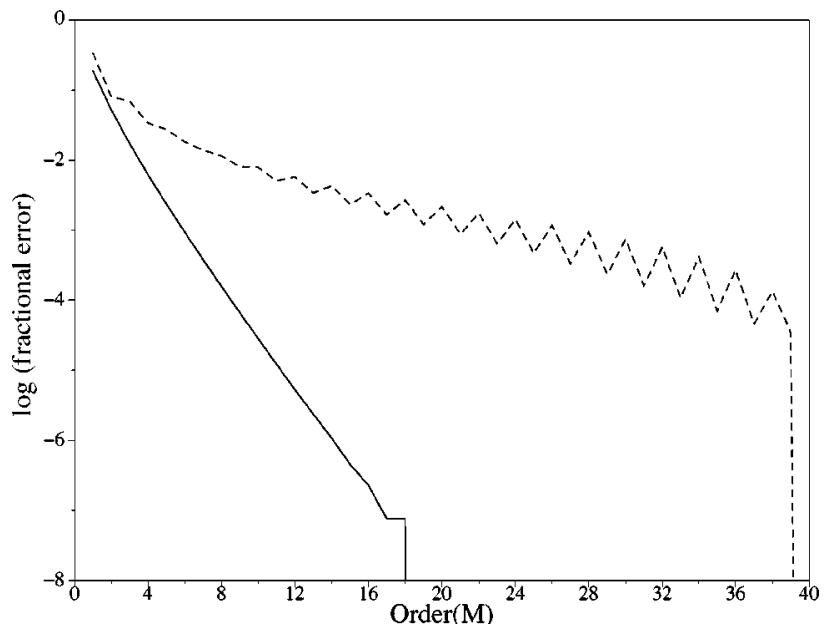

FIG. 1. Decimal logarithm of the fractional error $\left(E_{\text {app }} / E_{\text {ex }}-1\right)$ for the eigenvalue $m=+20$ of the Hamiltonian, Eq. (14), using $N_{\text {tot }}=81$ grid points between 0 and $2 \pi$ : FD approximation (solid line); truncation of the Fourier cardinal basis (dashed line). We used the parameters $\mu=1, r=1$ in a.u., and the order $M$ corresponds to stencils with $N=2 M+1$ grid points.

points. For comparison in Fig. 1 we plot the result of truncating the Fourier cardinal basis (dashed line). This means that we do not use the total set of grid points $\left(N_{\text {tot }}\right)$ to represent the wave function but a subset of $N$, which is also the size of the stencil in FD. We employed a grid of $N_{\text {tot }}=81$ points, so that the first $m=40$ eigenvalues are exactly calculated (notice that they are doubly degenerate). The important point here is that we can accurately represent an eigenvalue in the medium range of the spectrum by using significantly less grid points than the required in the pseudospectral approximation. Truncation of the PS series with even only a few points less than the full series results in significant errors, but we need only half of the grid points for the exact solution with FD.

\section{B. Periodic nonuniform grids}

In Hamiltonians describing vibrational motions of molecules, it is customary to find the polar angle dependent operator as

$$
-\frac{\hbar^{2}}{2 \mu r^{2}}\left(\frac{d^{2}}{d \theta^{2}}+\cot \theta \frac{d}{d \theta}\right),
$$

with $0 \leqslant \theta<\pi$. There have been several adaptations of the Fourier-PS method for this particular operator, ${ }^{24,25}$ however it is not very efficient since the singularities at $\theta=0$ and $\pi$ can cause numerical instabilities. For instance, one usually has to resort to shifting and symmetrically extending the grid around $\theta=\pi,{ }^{24,25}$ and constructing explicitly unitary propagators for stable propagation in time. The instability comes also from the fact that the transformation, Eq. (7), is not unitary in the polar angle $\theta$ for Fourier functions. ${ }^{26}$

The optimum DVR methods to be used with this operator are those which employ trigonometric orthogonal polynomials of Legendre- and Chebyshev-type as the finite basis representation. Since the argument of these polynomials is $x=\cos \theta$, the Hamiltonian operator takes the form

$$
-\frac{\hbar^{2}}{2 \mu r^{2}}\left[\left(1-x^{2}\right) \frac{d^{2}}{d x^{2}}-2 x \frac{d}{d x}\right],
$$

and the singularity is eliminated.

Using an orthogonal polynomial basis in Eqs. (6) and (7), $\phi_{k}=P_{k}$, to obtain the cardinal basis functions, we take

$$
u_{j}(x)=w_{j} \sum_{k=1}^{N} P_{k}\left(x_{j}\right) P_{k}(x), \quad j=1, \ldots N .
$$

$x_{j}$ are the zeros of the polynomial $P_{N+1}$ of degree $N$. Notice, that in Eq. (19), the summation starts from 1 which corresponds to the constant term in the polynomial. Hence, $P_{N}$ denotes a polynomial of degree $N-1$. The $\delta$-Kronecker property of the cardinal functions is satisfied by the Christoffel-Darboux theorem for orthogonal polynomials. $^{7,27}$ To show that the FD formulas are also the limit of orthogonal polynomial expansions in DVR methods, we have to establish the equivalence between the DVR functions, Eq. (19), and the Lagrange fundamental polynomials, $L_{j}(x)$, of order $N-1$.

First we note, that by the definition of Gaussian quadrature, $L_{j}(x)$ satisfy the orthogonality property

$$
\int_{a}^{b} L_{i}(x) L_{j}(x) d x=w_{j} \delta_{i j}, \quad i, j=1,2, \ldots, N,
$$

if they are evaluated at the zeros of some orthogonal polynomial. Then, an expansion of a function $F(x)$ in orthogonal polynomials can be represented with the series

$$
F(x) \approx \sum_{k=1}^{N} F_{k} P_{k}(x)
$$

where the coefficients are

$$
F_{k}=\int_{a}^{b} F(x) P_{k}(x) d x,
$$

and therefore, $F(x)$ can be defined through the integral

$$
F(x)=\int_{a}^{b} F\left(x_{j}\right) K_{N}\left(x_{j}, x\right) d x_{j} .
$$

The kernel $K_{N}\left(x_{j}, x\right)$ is defined by

$$
K_{N}\left(x_{j}, x\right)=\sum_{k=1}^{N} P_{k}\left(x_{j}\right) P_{k}(x) .
$$

On the other hand, expanding $F(x)$ in terms of Lagrange fundamental polynomials, Eqs. (1) and (2), we obtain

$$
\begin{aligned}
F(x) & =\sum_{k=1}^{N} F\left(x_{k}\right) L_{k}(x) \\
& =\int_{a}^{b} F\left(x_{j}\right) w_{j}^{-1} \sum_{k=1}^{N} L_{k}\left(x_{j}\right) L_{k}(x) d x_{j},
\end{aligned}
$$

taking into account the orthogonality relation, Eq. (20). Since, the kernels in Eqs. (23) and (25) must coincide, we have that 


$$
L_{j}(x)=w_{j} \sum_{k=1}^{N} P_{k}\left(x_{j}\right) P_{k}(x)
$$

using the $\delta$-Kronecker property of $L_{j}(x)$. Hence, we have shown that when we use the $N$ roots of the $P_{N+1}$ polynomial as interpolating grid points, the Lagrange fundamental polynomials are the cardinal functions that correspond to the orthogonal polynomials $P_{k}(x), k=1, \ldots N$.

We experimented with several grid distributions to numerically solve the eigenvalue equation

$$
-\frac{\hbar^{2}}{2 \mu r^{2}}\left(\frac{d^{2}}{d \theta^{2}}+\cot \theta \frac{d}{d \theta}\right) \Psi=\frac{l(l+1)}{2 \mu r^{2}} \Psi .
$$

First, we tried a uniform grid distribution in $\theta$ by means of Fourier. Although, in this simple case with no potential energy the Fourier basis also gives the exact result [using slightly different functions than those in Eq. (11)], since now the interval in $\theta$ has periodicity $\pi$ (see the Appendix), the FD convergence is very poor due to the bad approximations at the borders of the grid, around $\theta=0$ and $\pi$. We can think of it as Lagrange polynomials in $\theta$ present "cusps" at the borders of the grid, but the true solutions (Legendre polynomials) are a series in $\cos \theta$, and therefore, have a smooth behavior around 0 and $\pi$. A much better representation is achieved if we make the transformation $x=\cos \theta$ as mentioned above. Notice, that still we can use a uniform grid in $\theta$, since, if we choose as grid points

$$
\theta_{i}=\frac{\pi\left(i-\frac{1}{2}\right)}{N},
$$

and then by making the change of the variable to $x$ [Hamiltonian Eq. (18) instead of Eq. (17)], we have a ChebyshevDVR method.

The FD implementation can be done in two ways: at the end of the interval, where we need to supply more grid points for calculating the weights than those at our disposal, we can use a mixed centered and one-sided FD scheme and keep the order constant along the grid. That is, we take centered FDs until we reach the edge of the grid where we apply one-sided stencils. The other possibility is to impose the periodic boundary conditions, Eqs. (16), in the calculation of FD weights.

In Fig. 2 we show the results for three eigenvalues ( $l$ $=10,20$, and 30) calculated with FD for several orders of approximation, $M$ th, with mixed centered and one-sided stencils defined on the total number of Legendre grid points $N_{\text {tot }}=41$ (the roots of the Legendre polynomial of 41 st degree). Truncated Legendre-DVR calculations of the $l=20$ eigenvalue are shown with a dashed line. We remark that there are no noticeable differences by using centered FD with periodic boundary conditions or mixed centered, onesided stencils. We can see the bad convergence for high wave numbers mentioned in the introduction, although the result with FD is much better than truncation for the low and medium eigenvalues. In Fig. 3 we compare the FD calculations with a Chebyshev grid and periodic boundary condi-

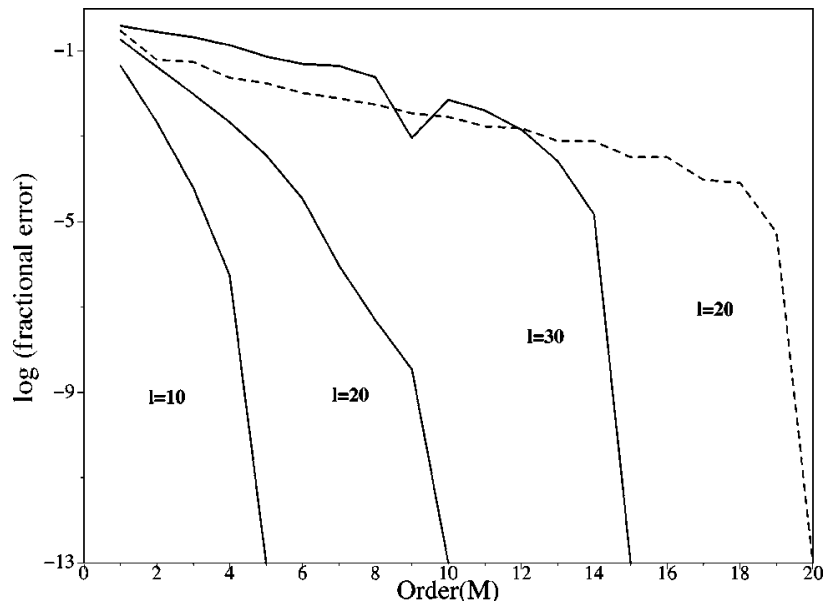

FIG. 2. Decimal logarithm of the fractional error for the eigenvalues $l$ $=10,20$, and 30 of the Hamiltonian, Eq. (27) using FD approximations of different order in a Legendre grid with mixed centered and one-sided stencils (solid lines). Also the Legendre-DVR result for $l=20$ using the basis functions, Eq. (19), is plotted with a dashed line for comparison. The total number of grid points used is $N_{\text {tot }}=41$.

tions (solid lines) with those of a Legendre grid and mixed centered, one-sided stencils (dashed lines). Practically, the results are the same.

\section{APPLICATION TO $\mathrm{SO}_{2}$}

To explore the performance of the above-described methods and the computer codes that we have developed in a realistic molecular potential, we have chosen a typical triatomic molecule, sulfur dioxide. Recently, extended threedimensional quantum mechanical calculations for the vibrational levels of this molecule were carried out by Guo and co-workers ${ }^{14-16}$ using the spectroscopic potential of Kauppi and Halonen. ${ }^{13}$ The potential is expressed in terms of the bend angle and Morse radial variables and reproduces the experimentally observed vibrational levels up to high energies. The characteristic of the new vibrational studies on $\mathrm{SO}_{2}$ is the size of the calculations. By applying filter diagonaliza-

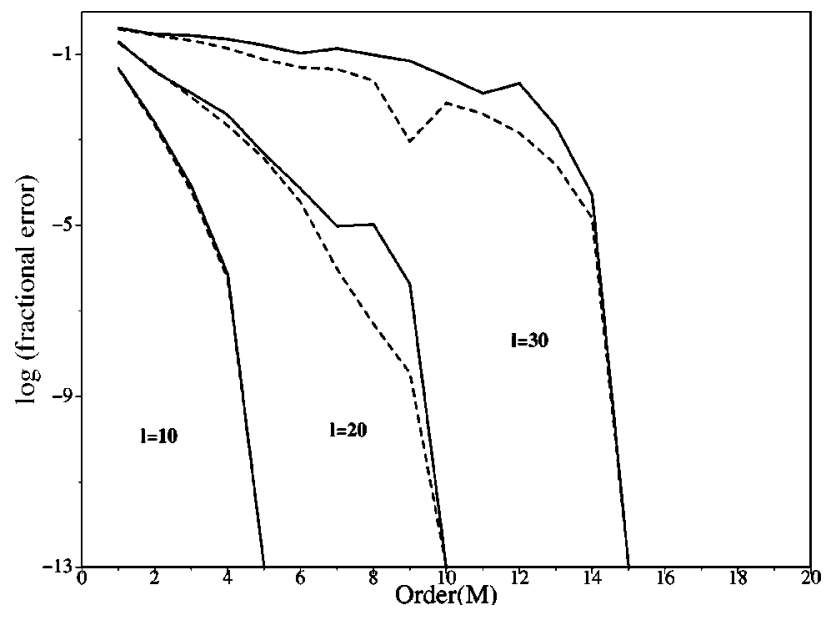

FIG. 3. The same as in Fig. 2 but using FD on a Chebyshev grid with periodic boundary conditions (solid lines). The results with a Legendre mixed centered, one-sided grid FD are plotted with dashed lines. 
tion algorithms, ${ }^{17-19}$ the researchers were able to compute about 5000 vibrational levels spanning an energy range up to $25000 \mathrm{~cm}^{-1}$. The operation, $\hat{H} \Psi$, was computed by discretizing the Radau coordinates in sinc-DVR for the two stretch modes and in a Legendre grid for the bend mode. Introducing symmetrized stretch coordinates the maximum grid was $(60 \times 60)$ points and for the angle 180 points. Statistical analysis of the energy level spacing distributions showed largely regular behavior. ${ }^{15,28-31}$

The purpose of our calculations is not to reproduce the eigenvalues of $\mathrm{Ma}$ and $\mathrm{Guo}^{15}$ but instead to propagate in time wave packets on the same potential function for testing the accuracy and stability of FD methods described in the previous section. Thus, we solve the time-dependent Schrödinger equation

$$
i \hbar \frac{\partial \Psi(t, \vec{q})}{\partial t}=\hat{H}(\vec{q}) \Psi(t, \vec{q}) .
$$

The vector $\vec{q}$ denotes, collectively, the Jacobi coordinates $R, r$, and $\theta$, which describe the internal motion of the molecule; $R$ is the distance from $\mathrm{S}$ to the center-of-mass of $\mathrm{O}_{2}$, $r$ is the distance between the two oxygen atoms, and $\theta$ the angle between $R$ and $r$ :

$$
\begin{aligned}
\hat{H}= & -\frac{\hbar^{2}}{2 \mu_{R}} \frac{\partial^{2}}{\partial R^{2}}-\frac{\hbar^{2}}{2 \mu_{r}} \frac{\partial^{2}}{\partial r^{2}} \\
& -\frac{\hbar^{2}}{2}\left(\frac{1}{\mu_{R} R^{2}}+\frac{1}{\mu_{r} r^{2}}\right)\left(\frac{\partial^{2}}{\partial \theta^{2}}+\cot \theta \frac{\partial}{\partial \theta}\right)+V(R, r, \theta),
\end{aligned}
$$

where, $\mu_{R}$ and $\mu_{r}$ are the reduced masses for $\mathrm{S}-\mathrm{O}_{2}$ and $\mathrm{OO}$, respectively.

The initial number of grid points for the three variables is $N_{\text {tot }}=64$ or 128 points in each dimension. The 128-point grids would require vectors of more than two million elements if cubic grids had to be used. However, taking into account that the wave function is zero at those grid points where the potential value is higher than a threshold, we store only a fraction of them, thus reducing substantially the size of the arrays. The energy cutoff was taken at $3 \mathrm{eV}$ but some results were checked with $4 \mathrm{eV}$ as well.

For the stretch $r$ coordinate, uniform equispaced grids are used. The energy to linearize the molecule is more than 4 $\mathrm{eV}$, higher than the energies we have considered. Thus, we assume that the wave function is zero at $R=0$ and apply equispaced uniform distributions for this coordinate. Nevertheless, tests with mixed centered, one-sided stencils are also examined. For the angle variable we take uniform, equispaced grids with zero boundary conditions as well as Chebyshev and Legendre grids with periodic boundary conditions.

The time-dependent Schrödinger equation was numerically solved using a Chebyshev expansion ${ }^{9}$ for the time propagator. Two initial Gaussian wave packets are chosen which we label (wp1), with center at $R=0.915 \AA$, $r=2.4768 \AA, \theta=90^{\circ}$, and potential energy $E=0.7480 \mathrm{eV}$, and the wave packet (wp2) with center $R=1.015 \AA$, $r=2.4768 \AA, \theta=90^{\circ}$, and potential energy $E=1.5708 \mathrm{eV}$. The widths for both Gaussians were $\sigma_{R}=0.05 \AA^{-1}$,
TABLE I. Conservation of the norm $\left(N_{t}\right)$ and total energy $(E)$ for several order finite difference approximations during the propagation of wave packet wp1 (see text). The grid distributions in $(\theta, R, r)$ are denoted with the symbols, (u) for centered, equispaced stencils, (m) for mixed centered and one-sided stencils, (1) for Legendre, and (c) for Chebyshev grid points. The imaginary part of the energy is the value at the end of the iterations. The iterations are counted by the total number of $\hat{H} \Psi$ operations. The total run times are $1.14 \mathrm{ps}$ for the grids of 64 points and 2.03 ps for the grids of 128 points. The numbers in parentheses are the power of ten.

\begin{tabular}{lcccccr}
\hline Method & Grid & Points & $\frac{N_{t}}{N_{0}}-1^{\mathrm{a}}$ & $\frac{E}{E_{0}}-1^{\mathrm{b}}$ & Imaginary & \multicolumn{1}{c}{$\hat{H} \Psi$} \\
\hline FFT & uuu & 64 & $1.4(-3)$ & $1.7(-3)$ & $3.8(-6)$ & 342016 \\
FD-2 & cuu & 64 & $1.0(-7)$ & $1.5(-7)$ & $1.7(-9)$ & 90112 \\
FD-4 & luu & 64 & $1.0(-7)$ & $1.1(-7)$ & $-1.5(-9)$ & 90112 \\
FD-7 & cuu & 64 & $1.0(-8)$ & $5.3(-8)$ & $-3.6(-10)$ & 61440 \\
FD-12 & cuu & 64 & $1.0(-8)$ & $4.2(-8)$ & $-1.1(-9)$ & 61440 \\
FD-2 & uuu & 128 & $1.0(-8)$ & $1.0(-8)$ & $-4.4(-13)^{\mathrm{c}}$ & 538624 \\
FD-2 & cuu & 128 & $1.0(-8)$ & $2.1(-8)$ & $1.8(-10)$ & 223232 \\
FD-4 & cuu & 128 & $1.0(-8)$ & $1.1(-8)$ & $6.2(-11)$ & 221184 \\
\hline \hline
\end{tabular}

${ }^{\mathrm{a}} N_{0}$ is the norm at time $t=0$, and $N_{t}$ the norm at the end of iterations.

${ }^{\mathrm{b}} E_{0}$ is the total energy at time $t=0$, and $E$ the energy at the end of iterations.

${ }^{c}$ The accuracy of the norm and energy is higher than $1.0 \times 10^{-8}$ which is printed out.

$\sigma_{r}=0.05 \AA^{-1}$, and $\sigma_{\theta}=0.08 \mathrm{rad}^{-1}$. Both wave packets are symmetric with respect to the line $\theta=90^{\circ}$ and with the $\mathrm{OO}$ bond at its equilibrium value. Hence, we expect excitation of the symmetric stretch and bend states but no excitation of the antisymmetric states.

From the time propagation of the initial wave packet we calculate the autocorrelation function, $C(t)$ $=\langle\Psi(0, \vec{q}) \mid \Psi(t, \vec{q})\rangle$, the Fourier transform of which reveals the eigenstates which overlap with the initial wave packet. The stability in the time propagation was checked by the norm of the wave packet, which should be one for an initial normalized function, and the conservation of the total energy. Both quantities should be conserved for bound motions. The imaginary part of the energy was also printed out as an indicator of the stability and accuracy of the calculation.

Calculations have been performed with variable order FD and several grid distributions which are denoted with the symbols, (u) for centered, equispaced stencils, (m) for mixed centered and one-sided stencils, (1) for Legendre, and (c) for Chebyshev grid points. Thus, the triad (luu), for example, denotes that Legendre points were used for the bend coordinate and centered, equispaced grids for the $R$ and $r$ stretch coordinates, respectively. All calculations were performed with PCs of $450 \mathrm{MHz}$ and 512 MBytes memory.

In Tables I and II we show the relative errors in the norm and energy after propagating the wave packets, wp1 and wp2, respectively. The time evolution was for 1.14 or 2.03 ps. FFT calculations are also presented for a cubic grid with 64 points in each dimension. From Tables I and II we can see that FFT preserves the norm and the energy at the third decimal point. In the finite difference approximation, the order $M$ corresponds to stencils with $N=2 M+1$ points. We find no significant differences between Chebyshev and Legendre grids for the angle variable. Increasing the number of grid 
TABLE II. Conservation of the norm $\left(N_{t}\right)$ and total energy $(E)$ for several order finite difference approximations during the propagation of wave packet wp2 (see text). The grid distributions in $(\theta, R, r)$ are denoted with the symbols, (u) for centered, equispaced stencils, $(\mathrm{m})$ for mixed centered and one-sided stencils, (l) for Legendre, and (c) for Chebyshev grid points. The imaginary part of the energy is the value at the end of the iterations. The iterations are counted by the total number of $\hat{H} \Psi$ operations. The total run times are $1.14 \mathrm{ps}$. The numbers in parentheses are the power of ten.

\begin{tabular}{lcccccr}
\hline \hline Method & Grid & Points & $\frac{N_{t}}{N_{0}}-1^{\mathrm{a}}$ & $\frac{E}{E_{0}}-1^{\mathrm{b}}$ & Imaginary & $\hat{H} \Psi$ \\
& & & & & & \\
\hline FFT & uuu & 64 & $1.3(-3)$ & $1.4(-3)$ & $1.9(-6)$ & 260096 \\
FD-2 & cmu & 64 & $1.0(-8)$ & $1.0(-8)$ & $-8.3(-10)$ & 59392 \\
FD-4 & cmu & 64 & $1.0(-8)$ & $5.8(-8)$ & $4.0(-10)$ & 59392 \\
FD-7 & uuu & 64 & $1.0(-8)$ & $1.0(-8)$ & $2.7(-13)^{\mathrm{c}}$ & 153600 \\
FD-7 & cuu & 64 & $1.0(-8)$ & $1.0(-8)$ & $6.7(-11)^{\mathrm{c}}$ & 61440 \\
FD-9 & cuu & 64 & $1.0(-8)$ & $1.0(-8)$ & $3.4(-10)^{\mathrm{c}}$ & 61440 \\
FD-9 & luu & 64 & $1.0(-8)$ & $1.0(-8)$ & $-5.5(-11)^{\mathrm{c}}$ & 61440 \\
FD-2 & cuu & 128 & $1.0(-8)$ & $1.0(-8)$ & $-3.2(-11)^{\mathrm{c}}$ & 159744 \\
FD-7 & cuu & 128 & $1.0(-8)$ & $1.0(-8)$ & $3.1(-12)^{\mathrm{c}}$ & 157696 \\
\hline \hline
\end{tabular}

${ }^{\mathrm{a}} N_{0}$ is the norm at time $t=0$, and $N_{t}$ the norm at the end of iterations.

${ }^{\mathrm{b}} E_{0}$ is the total energy at time $t=0$, and $E$ the energy at the end of iterations.

${ }^{\mathrm{c}}$ The accuracy of the norm and energy is higher than $1.0 \times 10^{-8}$ which is printed out.

points to 128 , the imaginary part of the energy decreases even for low order approximations. As has been shown in Paper I, higher accuracies are achieved either by increasing the order of approximation or the number of grid points. Finally, we point out that mixed centered and one-sided grids lead to larger instabilities as the order of approximation increases. Taking a uniform grid for the angle in a smaller interval than $[0, \pi]$ we obtain a representation of the wave function with more grid points, and that explains the large number of $\hat{H} \Psi$ operations for the (uuu) grids in Tables I and II. Overall, we have found that centered equispaced grids give the most stable and accurate results.

Part of the spectrum is shown in Fig. 4. The dashed lines correspond to the eigenenergies published by Ma and Guo ${ }^{15}$

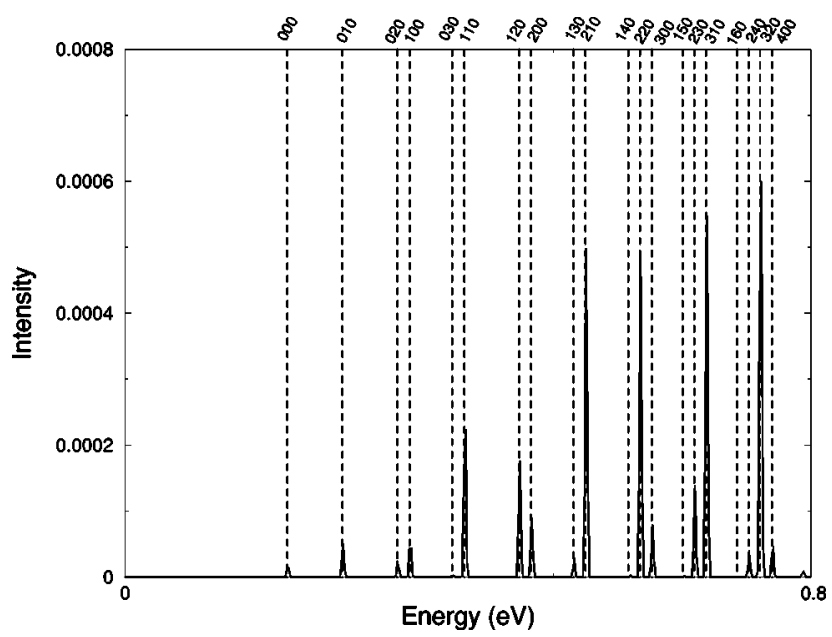

FIG. 4. Comparison of some eigenenergies obtained by Ma and Guo (Ref. 15) (dashed lines) and finite difference at second-order on a (uuu) grid and 128 initial points for each coordinate. The numbers at the top denote the quantum numbers for the the $R$-stretch, bend, and $r$-stretch, respectively.

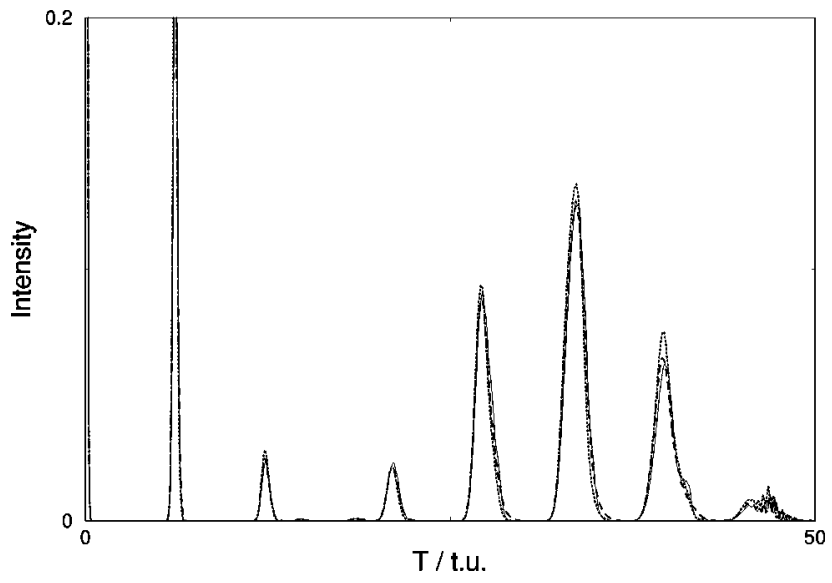

FIG. 5. Correlation functions calculated with the initial wave packet wp2 at second-order FD approximation on a (cuu) grid and 128 initial points (solid line), seventh-order FD with a 64 (cuu) initial grid (dashed line), and the FFT on a 64 orthogonal grid (dotted line). The time unit is 0.01018044 ps.

and the labels at the top of the figure are the assigned quantum numbers for the $R$-stretch, bend, and $r$-stretch, respectively. The spectrum was obtained with the wave packet wp1, and with a second-order FD on a (uuu) grid of 128 initial points for each coordinate. Obviously, techniques such as filter diagonalization must be applied for an accurate determination of the eigenlevels. Here, it is not our purpose to extract exact eigenvalues, but rather to investigate the stability of finite difference approximations in the time evolution of wave packets. It should be emphasized that symmetry considerations which speed up ordinary DVR calculations could also be applied in FD methods.

In Fig. 5 we compare the correlation functions of the wp2 wave packet calculated with second-order FD on a (cuu) grid and 128 initial points (solid line), seventh-order FD with a 64 (cuu) initial grid (dashed line), and the FFT on a 64 orthogonal grid (dotted line).

In Fig. 6 we compare the power spectra in the energy interval of 1.5 to $1.7 \mathrm{eV}$ obtained after propagating the wp2 for 4.17 ps and using fourth- (dotted line), fifth- (dashed

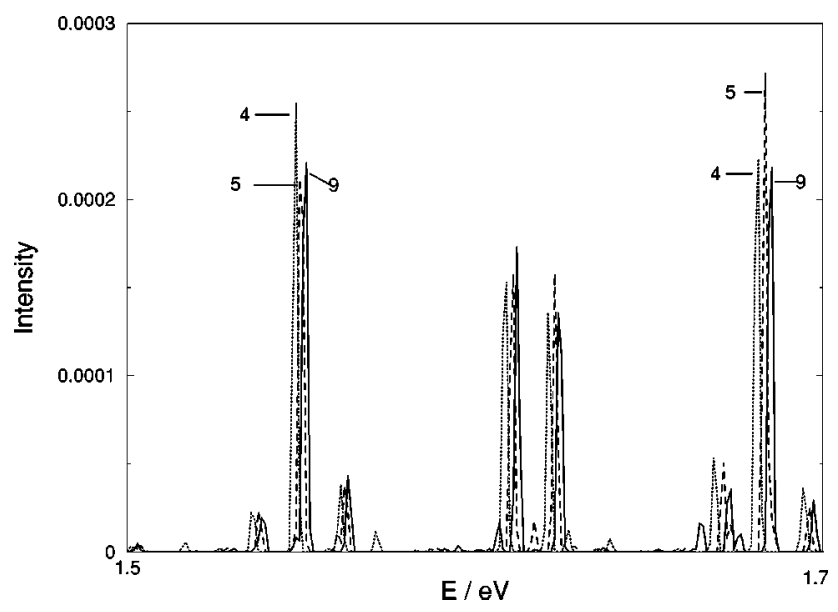

FIG. 6. Convergence of the power spectra obtained with wave packet wp2 and fourth- (dotted line), fifth- (dashed line), and ninth- (solid line) order FD on a 64 (cuu) grid. Higher order approximations give peaks which coincide with that of ninth-order. 
TABLE III. The intensities of the four more intense peaks of Fig. 6 at high order FD approximations after propagating wp2 for $4.17 \mathrm{ps}$ on 64 (cuu) grid.

\begin{tabular}{lcccc}
\hline \hline Energy & FD-9 $\left(\times 10^{-3}\right)$ & FD-11 $\left(\times 10^{-3}\right)$ & FD-13 $\left(\times 10^{-3}\right)$ & FD-15 $\left(\times 10^{-3}\right)$ \\
\hline 1.55152 & 0.221029 & 0.232002 & 0.235093 & 0.236147 \\
1.61203 & 0.173382 & 0.169127 & 0.167414 & 0.166750 \\
1.62393 & 0.135979 & 0.127174 & 0.124286 & 0.123204 \\
1.68544 & 0.218233 & 0.237179 & 0.242576 & 0.244425 \\
\hline \hline
\end{tabular}

line), and ninth- (solid line) order FD on a 64 (cuu) grid. For higher order approximations the peaks have been converged within the Fourier transform resolution $(0.001 \mathrm{eV})$. In Table III we show how the intensity varies for four peaks by increasing further the order of approximation.

Finally, in Fig. 7 we plot the wave packet wp1 after integrating for 1.54 ps with second-, fourth-, seventh-, and twelfth-order FD approximations on a 64 (uuu) grid. The projection of the wave function on the $(\theta, R)$ plane integrated over the $r$ coordinate is shown. We can see, that for converged wave functions we must use high order FD.

\section{CONCLUSIONS}

Variable high order finite difference algorithms for calculating the derivatives in a molecular Hamiltonian have been studied and tested for stability and accuracy by solving the time-dependent and time-independent Schrödinger equation with angular variables. Relations of high order FD limits to Fourier and orthogonal polynomial discrete variable representations have been analytically established and numerically investigated. The importance of these limits is that, by using a single code to generate the FD weights, we can systematically explore a variety of different approximations ranging from low order FD to sinc, Fourier, and orthogonal polynomial DVR, simply giving different grid distributions as input and imposing the proper boundary conditions. Here, we have examined centered and mixed centered, one-sided uniform grids, as well as Chebyshev and Legendre periodic grids. From the applications we have made up to now we find that the centered equispaced grids give a good represen-

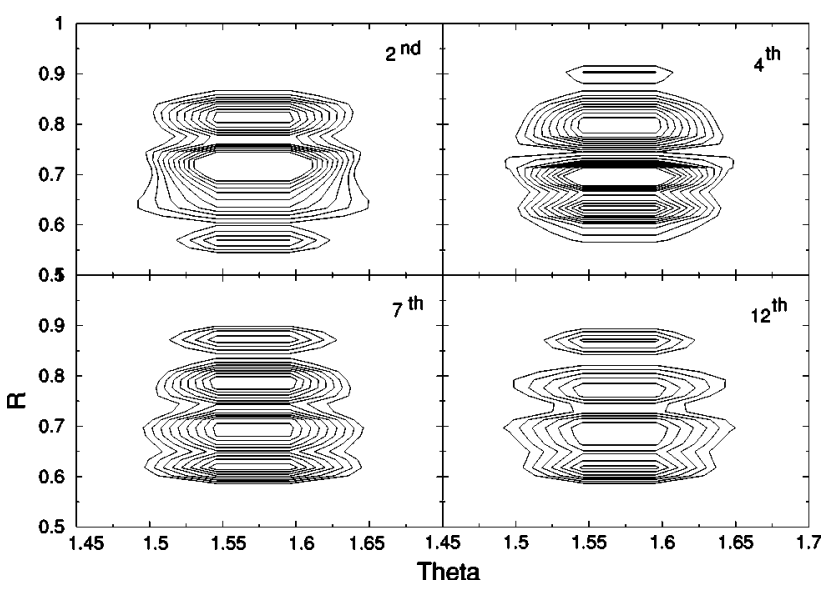

FIG. 7. Projections of the wave packet wp1 after integrating for $1.54 \mathrm{ps}$ with second-, fourth-, seventh-, and twelfth-order FD approximations. Distance in $\AA$ and angle in radians. tation of the wave function and result in stable time propagation. Particularly, for $\mathrm{SO}_{2}$ with a triangular equilibrium geometry, a uniform grid in a proper interval gives a better representation of the wave function than the Legendre or Chebyshev grid with points accumulated at the end of the interval. Finally, mixed centered, one-sided stencils of high order approximations lead to instabilities. We find practically the same results using either Legendre or Chebyshev grid points.

From Paper I and this work the following advantages of the FD approach in solving the Schrödinger equation emerge:

(1) finite differences allow a systematic search of the convergence properties with respect to the number of grid points as well as the order of approximation of the derivatives;

(2) we can use a large number of grid points for better representing the wave function and save computer time and memory by employing low order approximations;

(3) finite differences with stencils equal to the total number of grid points are equivalent to the most common PS methods (sinc, Fourier, Chebyshev, Legendre);

(4) truncated PS methods are generally bad approximations whereas finite differences show smooth convergence behavior by increasing the order;

(5) there is flexibility in choosing the grid points without necessarily any dependence on specific basis functions;

(6) algorithms for a fast generation of the weights in the FD approximations of the derivatives by recursion relations are available; and

(7) the computer codes can be parallelized.

Further work on tetratomic molecules is in progress.

\section{ACKNOWLEDGMENTS}

R. Guantes acknowledges financial support from a European Union TMR grant (FM-RX-CT-97-0101), and a postdoctoral grant from CAM, Spain. We thank Dr. Rita Prosmiti for useful discussions.

\section{APPENDIX}

For completeness of the article we provide here the coefficients $b_{k, j}^{(m)}=d^{m} u_{j}(x) /\left.d x^{m}\right|_{x=x_{k}}$ for obtaining the first $(m=1)$ and second $(m=2)$ derivatives of the Fourier cardinal basis functions $u_{j}(x)$ [Eq. (11)] and with periodicity $L$. Similar equations were first extracted by Meyer $^{32}$ and later on by Colbert and Miller: ${ }^{5}$ 


$$
b_{k, j}^{(1)}=\left\{\begin{array}{ll}
\frac{2 \pi}{L}(-1)^{l} /(2 \sin (l \pi / N)) & l= \pm 1, \pm 2, \ldots \\
& ( \pm 1 \pm N),( \pm 2 \pm N) \ldots \text { periodically extended } \\
0 & l=0, \pm N, \pm 2 N, \ldots
\end{array}\right\}
$$

with $l=j-k$, and

$$
b_{k, j}^{(2)}=\left\{\begin{array}{ll}
\left(\frac{2 \pi}{L}\right)^{2}(-1)^{l+1} \cos (l \pi / N) /\left(2 \sin ^{2}(l \pi / N)\right) & l= \pm 1, \pm 2, \ldots \\
\left(\frac{2 \pi}{L}\right)^{2} \frac{1}{12}\left(1-N^{2}\right) & ( \pm 1 \pm N),( \pm 2 \pm N) \ldots \\
& l=0, \pm N, \pm 2 N, \ldots
\end{array}\right\} .
$$

${ }^{1}$ R. Guantes and S. C. Farantos, J. Chem. Phys. 111, 10827 (1999).

${ }^{2}$ R. Guantes, A. Nezis, and S. C. Farantos, J. Chem. Phys. 111, 10836 (1999).

${ }^{3}$ B. Fornberg, in A Practical Guide to Pseudospectral Methods, Cambridge Monographs on Applied and Computational Mathematics (Cambridge University Press, Cambridge, England, 1998), Vol. 1; B. Fornberg and D. M. Sloan, Acta Numerica (Cambridge University Press, Cambridge, England, 1994), Vol. 3, pp. 203-267.

${ }^{4}$ J. P. Boyd, J. Comput. Phys. 103, 243 (1992).

${ }^{5}$ D. T. Colbert and W. H. Miller, J. Chem. Phys. 96, 1982 (1992).

${ }^{6}$ W. H. Press, B. P. Flannery, S. A. Teukolsky, and W. T. Vetterling, Numerical Recipes (Cambridge University Press, Cambridge, England, 1986).

${ }^{7}$ A. S. Dickinson and P. R. Certain, J. Chem. Phys. 49, 4209 (1968); J. C. Light, I. P. Hamilton, and J. V. Lill, ibid. 82, 1400 (1985).

${ }^{8}$ R. Kosloff, in Dynamics of Molecules and Chemical Reactions, edited by R. E. Wyatt and J. Z. H. Zhang (Dekker, New York, 1996).

${ }^{9}$ R. Kosloff, Annu. Rev. Phys. Chem. 45, 145 (1994).

${ }^{10}$ M. Thachuk and G. C. Schatz, J. Chem. Phys. 97, 7297 (1992).

${ }^{11}$ D. A. Mazziotti, Chem. Phys. Lett. 299, 473 (1999).

${ }^{12}$ G. W. Wei, D. S. Zhang, D. J. Kouri, and D. K. Hoffman, Phys. Rev. Lett. 79, 775 (1997).

${ }^{13}$ E. Kauppi and L. Halonen, J. Chem. Phys. 96, 2933 (1992).

${ }^{14}$ G. Ma, R. Chen, and H. Guo, J. Chem. Phys. 110, 8408 (1999).
${ }^{15}$ G. Ma and H. Guo, J. Chem. Phys. 111, 4032 (1999).

${ }^{16}$ D. Xie, G. Ma, and H. Guo, J. Chem. Phys. 111, 7782 (1999).

${ }^{17}$ V. A. Mandelshtam and H. S. Taylor, J. Chem. Phys. 106, 5085 (1997).

${ }^{18}$ V. A. Mandelshtam and H. S. Taylor, J. Chem. Phys. 107, 6756 (1997).

${ }^{19}$ R. Chen and H. Guo, Chem. Phys. Lett. 279, 252 (1997).

${ }^{20}$ J. T. Muckerman, Chem. Phys. Lett. 173, 200 (1990).

${ }^{21}$ D. Baye and P-H. Heenen, J. Phys. A 19, 2041 (1986); H. Karabulut and E. L. Sibert III, J. Phys. B 30, L513 (1997).

${ }^{22}$ I. S. Gradshteyn and I. M. Ryzhik, Table of Integrals, Series, and Products (Academic, 1994), formula 1.4223.

${ }^{23}$ B. Fornberg, Math. Comput. 51, 699 (1988).

${ }^{24}$ C. E. Dateo, V. Engel, R. Almeida, and H. Metiu, Comput. Phys. Commun. 63, 435 (1991); C. E. Dateo and H. Metiu, J. Chem. Phys. 95, 7392 (1991).

${ }^{25}$ R. C. Mowrey, Y. Sun, and D. J. Kouri, J. Chem. Phys. 91, 6519 (1989).

${ }^{26}$ G. C. Corey and D. Lemoine, J. Chem. Phys. 97, 4115 (1992).

${ }^{27}$ G. Szegö, Orthogonal Polynomials (American Mathematical Society Colloquium, New York, 1948), Vol. 23, Sec. 3.2, p. 42.

${ }^{28}$ S. C. Farantos, Laser Chem. 13, 87 (1993).

${ }^{29}$ R. Prosmiti, S. C. Farantos, and H. S. Taylor, Mol. Phys. 82, 1213 (1994).

${ }^{30}$ T. Weston and M. S. Child, Chem. Phys. Lett. 262, 751 (1996).

${ }^{31}$ R. Prosmiti, S. C. Farantos, and H. Guo, Chem. Phys. Lett. 311, 241 (1999).

${ }^{32}$ R. Meyer, J. Chem. Phys. 52, 2053 (1970). 